\title{
Some Systematic Features in the Distribution of Stars.
}

\section{By Fredericr H. Seares, Mount Wilson Observatory, Pa sadena, California.}

$T^{\mathrm{H}}$ HE mean distribution of the stars with respect to magnitude and galactic latitude derived by Prof. van Rhijn, Miss Joyner, Miss Richmond and myself, ${ }^{1}$ is expressed through values of $\log N_{m} \quad\left(N_{m}=\right.$ number of stars per sq. deg. brighter than $m$ ) which for the same latitude, north and south, are the same in all galactic longitudes. The supposition that densities having this characteristic may represent the observed features of stellar distribution assumes the sun to be at the centre of a stellar system having rotational symmetry. Although sufficiently exact for many purposes, this assumption is only a first approximation to the state of affairs in the heavens.

To obtain a further approximation, we may examine the deviations from the mean distribution observed in many parts of the sky. A discussion of the available data by Miss Joyner and myself reveals a conspicuous periodic irregularity extending into high latitudes, which, next to the galactic concentration, is perhaps the most striking feature of the distribution of stars over the sky.

With some additions which need not be detailed here, we have used the same observational material as for the derivation of the mean distribution, namely, the Mount Wilson Catalogue of Selected Areas, the Harvard-Groningen Durchmusterung, and counts from zones of the. Astrographic Catalogue published by Turner. The data from the last two sources were reduced to the international photographic scale with the aid of the Mount Wilson Catalogue and the mean distribution table.

Values of the deviation $\Delta$ have, in general, been calculated for $10^{\circ}$ intervals in longitude and latitude, from $70^{\circ} \mathrm{N}$. to $70^{\circ} \mathrm{S}$., for each of the limiting magnitudes $9,11,13.5,16$, and 18 . An important exception occurs only in the case of declinations south of $-15^{\circ}$, where, for the faint stars, we have only the data from the HarvardGronigen Durchmusterung, with a limiting magnitude of $16 \cdot 86$. Since the systematic change in $\Delta$ with magnitude is slow, we have assumed that the deviations found from this catalogue might be taken as representative of those for the limits $m=16$ and 18 and used with data for these limits from other parts of the sky.

The values of $\Delta$ for the same limiting magnitude and the same latitude show in all cases a variation with longitude which can be represented by a simple cosine term. Other irregularities are of course present, notably those corresponding to the obscuration in Taurus and Ophiuchus and to the abrupt changes in density in and near the Milky Way; but underneath all these the large-scale, periodic fluctuation is easily discerned. In spite of some progression, the longitudes of maximum deviation are much the same for all magnitudes and in all latitudes. Further, the amplitude of the variation decreases with increasing distance from the Milky

1 Cf. Nature, 115, 948 ; 1925 ; Mt. Wilson Contributions, No. 301 ; Astrophysical Journat, 62, $320 ; 1925$.
Way; and, finally, the constant term in $\Delta$ is always small, which shows that the asymmetry between northern and southern galactic hemispheres is slight.

In a general way these are the characteristics to be expected from an eccentrically located sun, only slightly removed from the galactic plane. Certain systematic differences in the deviations for the two hemispheres suggest, however, the probability of an error in the adopted position of the galactic pole, which is that of Gould. The mean distribution table, although based on data referred to a possibly erroneous pole, is little affected by such an error. The combination of observed densities from all longitudes made in forming this table eliminates the first-order terms in the error; but when observed densities are compared individually with the mean distribution, the error appears in the deviation $\Delta$.

We were thus led to the equation of condition.

$$
\Delta=s+G+F \cos (\lambda-L) \mp k \cos \left(\lambda-L_{o}\right),
$$

in which the upper sign refers to northern latitudes, the lower to southern; $s$ provides for a possible systematic correction to the mean distribution table; $k$ depends on $p$, the polar distance of the true galactic pole, in longitude $L_{o}$, relative to the adopted pole.

The use of this equation only removes the restriction that the sun should be centrally located among the stars, the original assumption of rotational symmetry being tacitly retained. Starting from this modified assumption, we interpret $L$ as the longitude of the centre of the stellar system, and $F$ and $G$ as functions of the latitude, the spacedensity, and the rectangular co-ordinates of the centre of the system relative to the sun. Although sufficient for present requirements, the equation is correct only to quantities of the first order in $p$ and $\rho / R$, where $\rho$ is the distance of the sun from the centre and $R$ the radius of the system.

Values of the parameters were calculated for each pair of latitudes, north and south, at $0^{\circ}, 5^{\circ}$, $10^{\circ}, 20^{\circ}, 30^{\circ} \ldots 70^{\circ}$, and where possible, combined into means which are collected in the accompanying table. Only $s$, which is of no interest here, has

\begin{tabular}{|r|c|c|c|c|c|}
\hline$m$. & $L$. & $L_{o}$ & $p$. & $2 F$. & $2 G$. \\
\cline { 1 - 3 } 9.0 & $267^{\circ}$ & $275^{\circ}$ & $8^{\circ} \cdot 1$ & 0.380 & -0.127 \\
11.0 & 270 & 296 & $6 \cdot 8$ & 0.434 & -0.101 \\
13.5 & 275 & 319 & 8.0 & 0.429 & -0.095 \\
16.0 & 319 & 357 & $4 \cdot 1$ & 0.690 & -0.012 \\
18.0 & 319 & 350 & 2.7 & 0.668 & +0.002 \\
\hline
\end{tabular}

been omitted. For $L, L_{o}$, and $p$, the average deviations for results from a single pair of latitudes are $7^{\circ} \cdot 0,10^{\circ} \cdot 4$, and $1^{\circ} \cdot 5$, respectively. The internal agreement therefore indicates uncertainties in the tabulated means of the order of $2^{\circ}$ to $4^{\circ}$ for $L$ and $L_{o}$, and of something less than $1^{\circ}$ for $p$; but N 2 
residual systematic errors in the data increase these limits considerably; the actual errors in the means are probably of the order of the average deviations quoted.

The significant result is that zones in all latitudes up to $70^{\circ}$ show the periodic irregularity of distribution corresponding to the foregoing formula, and give accordant values for the longitude of the centre of the system and the position of the galactic pole. Further, the errors of the respective means are such as to leave the obvious dependence of these quantities upon limiting magnitude open to no serious doubt.

The quantities $F$ and $G$, as already noted, are functions of the latitude, the space-density, and the distance of the sun from the centre of the system. We should like to utilise their values from all latitudes in a determination of the position of the sun. Substitution of the correct function logarithm of the ratio of the number of stars in longitude $L$ to the number in longitude $180^{\circ}+L$. This ratio also depends on the limiting magnitude, increasing from about, 2.5 for $m=9$ to app oximately 5 for $m=18$. An average of 4 , combined with the density law of Kapteyn and van Rhijn (which requires revision, and, moreover, is not strictly applicable), leads to a value of 1200 parsecs for the distance of the sun from the centre of the stellar system. This estimate neglects certain obvious refinements, but nevertheless must be of the right order of magnitude. It is so much smaller than Shapley's value of 25,000 parsecs for the distance from the centre of the svitem of globular clusters, that we may say at once that this system cannot be concentric with the general system of stars now within reach of our telescopes. It is well to remember, however, that the centres of both systems-of clusters and of stars-have

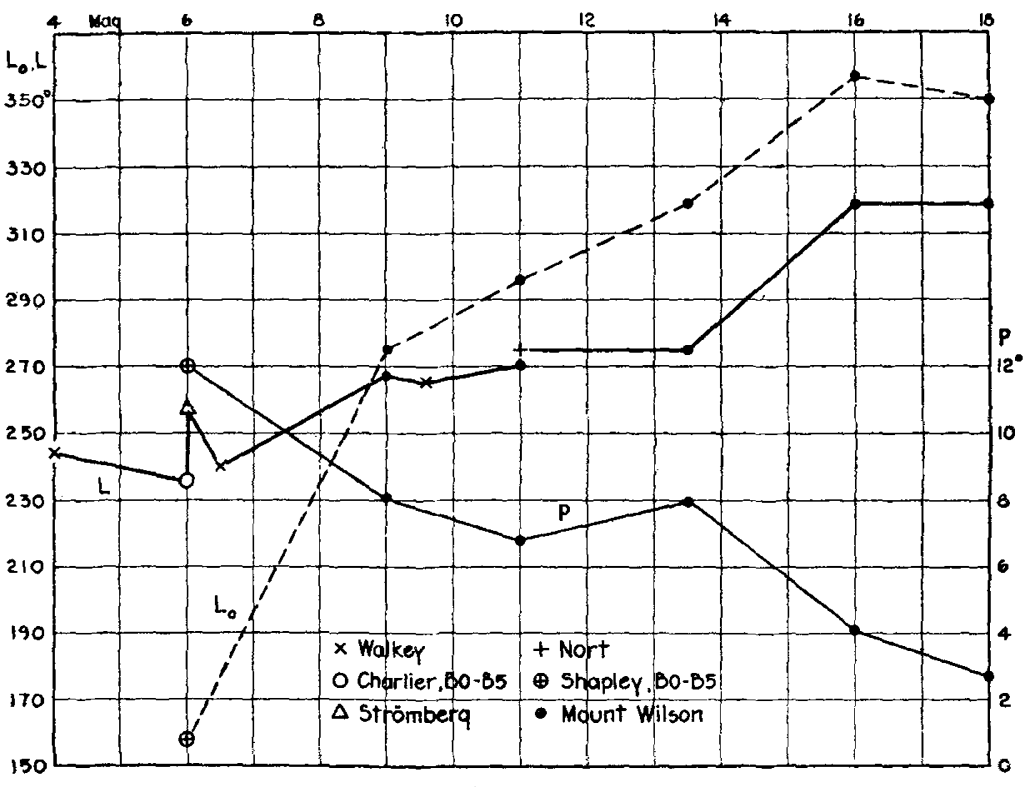

FIG. 1.

practically the same longitude.

The values of $G$ change so little that it seems permissible to tabulate means for all latitudes as an indication of the small displacement of the sun from the galactic plane. A value of $2 G$ equal to $0 \cdot 10$ for all limiting magnitudes would correspond to a distance of some 30 parsecs. Actually, however, the asymmetry is appreciable only for stars brighter than the fourteenth magnitude, which show an excess of about 25 per cent. in the southern latitudes. For the lower limiting magnitudes the difference between the two hemispheres is vanishingly small, and we may regard the sun as situated almost exactly in the corresponding galactic plane.

On the whole, we conclude that the systems of stars defined by successive limiting magnitudes differ appreciably in both centring and orientation. In this con-

for space-density and allowance for latitude should reduce all the values of $\vec{F}$ and $G$ for a given magnitude to constants, from which the co-ordinates of the centre could then be determined. Uncertain knowledge of the density function, together with the inherent complexity of the problem, blocks this direct method of procedure. The use of the simple but incorrect assumption of constant space-density leads to manageable formulæ, but leaves an uncompensated variation in $F$, and hence yields no very useful result. It does, however, prove again a well-known fact, for the residual variation in $F$ contradicts the assumption of constant density and shows that the stars thin out with increasing distance.

Because of the difficulty of utilising all the data, values of $F$ are given only for the galactic region, means for $0^{\circ}$ and $5^{\circ}$ being tabulated in order to reduce the influence of accidental error. Actually, the double value is given, since that quantity is the nexion results by other observers may be recalled. Walkey, Charlier, and Nort found the following longitudes of maximum density :

\begin{tabular}{cl} 
Limiting $m$. & \multicolumn{1}{c}{$L}$. \\
$4 \cdot 0$ & $244^{\circ}$ \\
$6 \cdot 5$ & 240 \\
$9 \cdot 6$ & 265 \\
$6 \pm$ & 236 (B0-B5 stars only) \\
11 & 275
\end{tabular}

From dynamical considerations of the characteristics of preferential motion $(m<6 \pm)$, Strömberg inferred that the centre of the system might be found in longitude $257^{\circ}$, while Shapley, from a study of globular clusters, adopted $326^{\circ}$. Further, Shapley has shown that the B0-B5 stars brighter than 5.5 lie close to a plane fixed by the pole having the co-ordinates $L_{o}=160^{\circ}, p=12^{\circ}$; while the nearer diffuse nebulæ, according to Hubble, define a 
'secondary galaxy' for which $L_{o}=160^{\circ}, p=20^{\circ}$. Other data and certain relevant comments might be added, but for the present account those quoted are sufficient.

The accompanying diagram (Fig. 1) shows the relation of all the results. Those just quoted both confirm and extend the conclusion derived from faint stars. In the systems defined by successive limits of brightness we find a continuous progression in the longitude of the centre and in the position of the galactic pole. The sequence seems to terminate with the galactic clouds; its upper end is the isolated group of helium stars known as the local cluster.

That the B0-B5 stars brighter than $5 \cdot 5$ constitute a local cluster seems clear. Most of these stars are within 500 parsecs and lie close to a plane inclined $12^{\circ}$ to the plane of the Milky Way. The fainter B's $(7 \cdot 26-8 \cdot 25)$, on the other hand, are more distant, and, as shown by Shapley and Miss Cannon, associated with the fundamental plane of the larger system. Direct evidence also connects some of the A stars with the local cluster. The present data, however, indicate that very faint stars, among them all spectral types, are included in its composition, or at least exhibit some of its characteristics. It is also evident that if the cluster is a separate dynamical unit, it must be much larger than hitherto supposed. Stars of the tenth to the fifteenth magnitude are certainly involved, because those brighter than the tenth magnitude are not numerous enough to influence appreciably the systems the limiting magnitudes of which are 12 or fainter. But these faint stars, which clearly show the progressive change in $L, L_{o}$, and $p$, extend to distances well beyond the most generously placed boundaries of the local system of $\mathrm{B}$ stars. It is perhaps questionable if the local cluster in this larger sense can be regarded as a separate unit ; the progressive change in the characteristics of stellar distribution is possibly analogous to that shown by Strömberg to be a striking feature of stellar motions.

\section{Fundamental Problems relating to River Pollution.}

\section{By H. W. Harvey.}

$\mathrm{T}^{\mathrm{H}}$ HE need for scientific research to provide a basis upon which to deal economically with the pollution of the rivers of Great Britain has been widely recognised during the last few years, and the Government is now prepared to provide adequate financial assistance for this purpose. It is of interest at the present moment to review the lines along which further research of a general character appears at first sight likely to prove most fruitful.

The types of pollution to which rivers and streams in England and Scotland are subject fall into two broad groups. In the first group fall inorganic compounds in the effluents from industrial undertakings, having a specific poisonous effect upon aquatic organisms. It may be taken that the chemist and engineer will find effective treatments for almost every such effluent, but their application will depend upon their cost; frequently the limited ground area available makes the simplest type of treatment, involving settling pits or the like, impracticable; frequently a partial treatment only is practicable, or necessary. In the latter case it is essential to know at what dilution the particular toxic substance will have a negligible effect upon various kinds of life natural to the river, for if the ordinary vegetable life in particular is seriously interfered with, slowrunning water may be depleted of dissolved oxygen and the natural processes of self-purification cease.

An inquiry into the normal seasonal changes in the aquatic life of an unpolluted stream is already in progress, with the final object of finding the effect of various types of pollution upon them, and of rendering it possible to gauge the extent to which any particular stream is harmfully polluted. It is recognised that considerable quantities of some solutes and matter in suspension may be run into a river without causing any material harm, and a mere change in the nature of the fauna does not provide sufficient ground for recommending the possibly costly treatment of effluents, provided that the capacity of the water to purify itself is not interfered with. This involves a biological research of some magnitude, and certainly of a fundamental nature.

Into the second group fall a wide and varied class of organic waste products. If these are turned into a river in suspension they settle upon and stifle the plant life, forming a substratum for fungus growth over the stones, or give rise to putrefaction, which may also happen if organic matter is added in solution. It is the event of putrefaction which opens up problems of the greater interest and the greater complication. As cases in point, the water in which beet is scrubbed at sugar factories, the water in which beans are boiled in American canning factories, and wash water from milk factories containing whey and a little milk, if run direct into a river in any quantity, rapidly deplete its waters of dissolved oxygen and kill off the animal and plant life in the stream, the latter being replaced by a fungus growth attached to the bottom. This is not the case with all organic matter, for I have met with cases where the effluent from treated sewage or even untreated sewage in small quantities appears, if anything, to improve the fishing in swift-running streams.

The question of why quite a limited amount of some organic substances should render a river foul and completely change the animal and vegetable community in it will repay most careful investigation. One general explanation is that putrefaction in the water, by using up the dissolved oxygen, renders it insufficiently aerated. In extreme cases the oxygen content of polluted rivers may fall to zero, as found in the esturine water at Newcastle during periods when little fresh water is coming down the Tyne ${ }^{\mathbf{1}}$; in other cases the absorption is

No. 2995, VoL. 119] 